\title{
Controlling of two destructive zooplanktonic predators in Chlorella mass culture with surfactants
}

\author{
Xiaobin Wen ${ }^{1,2}$, Aoqi Zhang 1,2,3, Xiaoyan Zhu ${ }^{1,2}$, Lin Liang ${ }^{3}$, Yan Huo ${ }^{1,2,3}$, Kaixuan Wang ${ }^{1,2,3}$, Youzhi Yu ${ }^{1,2,3}$, \\ Yahong Geng ${ }^{1,2}$, Yi Ding ${ }^{1,2^{*}}$ and Yeguang $\mathrm{Li}^{1,2^{*}}$
}

\begin{abstract}
Background: Predatory flagellates and ciliates are two common bio-contaminants which frequently cause biomass losses in Chlorella mass culture. Efficient and targeted ways are required to control these contaminations in Chlorella mass cultivation aiming for biofuel production especially.

Results: Five surfactants were tested for its ability to control bio-contaminations in Chlorella culture. All five surfactants were able to eliminate the contaminants at a proper concentration. Particularly the minimal effective concentrations of sodium dodecyl benzene sulfonate (SDBS) to completely eliminate Poterioochromonas sp. and Hemiurosomoida sp. were 8 and $10 \mathrm{mg} \mathrm{L}^{-1}$, respectively, yet the photosynthesis and viability of Chlorella was not significantly affected. These results were further validated in Chlorella mass cultures in 5, 20, and $200 \mathrm{~m}^{2}$ raceway ponds.

Conclusions: A chemical method using $10 \mathrm{mg} \mathrm{L}^{-1}$ SDBS as pesticide to control predatory flagellate or ciliate contamination in Chlorella mass culture was proposed. The method helps for a sustained microalgae biomass production and utilization, especially for biofuel production.
\end{abstract}

Keywords: Chlorella, Predator, Flagellates, Ciliates, Bio-contamination, Surfactant

\section{Background}

Chlorella is a genus of unicellular green microalgae that has long been used as a model organism to study photosynthesis [1]. Chlorella biomass is rich in protein, vitamins, and minerals. The success of Chlorella mass culture during the late 1940s created a stable Chlorella industry, primarily for human nutrition and animal feed [2, 3]. Recently, Chlorella is considered a candidate for bioenergy and bioremediation owing to its ability to grow fast, uptake nutrients in wastewaters, and synthesize a large amount of TAGs or carbohydrates in cells $[4,5]$.

\footnotetext{
*Correspondence: dingyi@wbgcas.cn; yeguang@wbgcas.cn

${ }^{1}$ Key Laboratory of Plant Germplasm Enhancement and Specialty

Agriculture, Wuhan Botanical Garden, Chinese Academy of Sciences, Wuhan 430074, China

Full list of author information is available at the end of the article
}

However, the current autotrophic technologies that are used for mass production of Chlorella biomass are facing challenges from biological contamination. Biological contamination occurs frequently in Chlorella mass culture in the widely used cultivation systems including circular and raceway ponds [3, 6-8]. Zooplanktonic predators, such as ciliates, rotifers, amoeba, and flagellates, are the most common contaminants as reported in the literature $[8,9]$. According to surveys by Ma et al. [10, 11], contamination by the predatory flagellate Poterioochromonas malhamensis in Chlorella culture can occur at any time throughout the year at any growing location. Ciliates are also widely spread and can cause serious problems to microalgae cultivation under broad climate conditions [12]. Invasion by these predators usually develops into bio-contamination. The most direct effect of such contamination is reduction of biomass yield. For example,

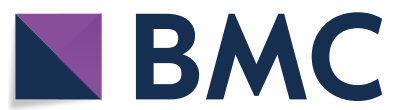

(c) The Author(s) 2021. This article is licensed under a Creative Commons Attribution 4.0 International License, which permits use, sharing, adaptation, distribution and reproduction in any medium or format, as long as you give appropriate credit to the original author(s) and the source, provide a link to the Creative Commons licence, and indicate if changes were made. The images or other third party material in this article are included in the article's Creative Commons licence, unless indicated otherwise in a credit line to the material. If material is not included in the article's Creative Commons licence and your intended use is not permitted by statutory regulation or exceeds the permitted use, you will need to obtain permission directly from the copyright holder. To view a copy of this licence, visit http://creativeco mmons.org/licenses/by/4.0/. The Creative Commons Public Domain Dedication waiver (http://creativecommons.org/publicdomain/ zero/1.0/) applies to the data made available in this article, unless otherwise stated in a credit line to the data. 
the cell density of Chlorella has been shown to decrease from $4.0 \times 10^{8}$ to $1.0 \times 10^{8}$ cells $\mathrm{mL}^{-1}$ within three days, whereas that of the grazer $P$. malhamensis increased to $1.1 \times 10^{6}$ cells $\mathrm{mL}^{-1}$ from $1.0 \times 10^{3}$ cells $\mathrm{mL}^{-1}$ [11] . Moreno-Garrido and Canavate [12] reported that grazing ciliates can visually clarify dense outdoor mass cultures of Dunaliella salina within 2 days. Similarly, over $60 \%$ of Chlorella biomass can be digested in a short time due to the explosive growth of predators such as flagellates or ciliates, according to the authors' experiences. Thus, the control of biological contamination is very important for the mass production of Chlorella in open systems.

Biological contaminations are different in their occurrence, development, and contamination mechanisms [6]. Many contaminations have occurred in an associative or sequential manner and interacted with the target microalgae [13]. These factors make the control of biological contamination very complicated. Methods have been suggested to overcome the challenges of biological contamination, such as filtration, changes of the environmental conditions such as medium $\mathrm{pH}$, and use of chemical additives including quinine, formaldehyde, ammonia, and hydrogen peroxide $[6,8,10,12]$. These methods are helpful in controlling different types of zooplanktonic contaminants. However, methods such as filtration and changes of medium $\mathrm{pH}$ are inefficient to apply in large scale, and some chemical additives, for example, ammonia and ammonium bicarbonate, are not applicable in microalgal cultivation where nitrogen limitation is necessary to induce TAG or astaxanthin accumulation since the addition of such chemicals will relieve nitrogen deficiency. Thus, more efficient and targeted ways are still required. Wang et al. [6] suggested that strain selection (non-susceptibility/resistance to biological pollutants) is the most practicable approach to cope with biological invasions, yet it is very time-consuming because a single algal species is unlikely to excel in all the required characteristics, such as resistance to biological pollutants, rapid growth, high product content, wide tolerance of environmental conditions, and other qualities that facilitate industrial production.

Surfactants (or 'surface active agents') are organic compounds that can modify the solution properties both within the bulk of the solution and at the solid/water interface [14], and they have been recognized as having certain cytotoxicity [15-17]. Cell membranes are the primary target for the toxicological effects of surfactants on cells, which are known to be loss of cell viability and cell lysis $[18,19]$. Here, we report on a simple and efficient chemical method, using surfactant as a single additive, to control the contamination of predatory flagellates and ciliates in Chlorella mass culture. Flagellates and ciliates, specifically Poterioochromonas sp. and Hemiurosomoida sp., have several similarities in the context of contamination in Chlorella mass culture in the present study. They are both unicellular, and can swim and graze on Chlorella cells and especially lack a resistant structure outside the plasma membrane [20-22] in comparison to Chlorella. These characteristics create possibilities for the targeted control of Poterioochromonas and Hemiurosomoida without inhibition on Chlorella growth. Five widely used surfactants in household cleaning products are used as pesticides and their effects on the two predators and Chlorella are investigated and compared. The application of this method is also discussed and recommended based on field testing.

\section{Results}

\section{Toxic effects of surfactants on predator growth} and reproduction

The successive transfer cultures of the two predators (Poterioochromonas sp. and Hemiurosomoida sp.) were established first as described in Methods. Using these successive transfer cultures, the efficacies of the five selected surfactants for controlling Poterioochromonas sp. and Hemiurosomoida sp. were evaluated. Toxic effects on both Poterioochromonas sp. and Hemiurosomoida sp. were observed for all five surfactants, namely sodium dodecyl benzene sulfonate (SDBS), coconut diethanolamide (CDEA), sodium dodecyl sulfate (SDS), fatty alcohol polyoxyethylene ether (AEO-7), and alcohol ethoxysulphate (AES).

As shown in Fig. 1, a greater than 30\% increase in cell densities of Poterioochromonas sp. were obtained after 24 h cultivation without surfactant addition, demonstrating the viability of the Poterioochromonas sp. cultures. However, the cell densities decreased in the cultures supplemented with any one of the five surfactants. For example, the cell density of the living Poterioochromonas sp. was $2.8 \times 10^{4}$ cells $\mathrm{mL}^{-1}$ in the culture without SDBS addition, yet it decreased to $1.8 \times 10^{4}$ cells $\mathrm{mL}^{-1}$ in the culture supplemented with $3 \mathrm{mg} \mathrm{L}^{-1}$ SDBS and further decreased to less than 100 cells $\mathrm{mL}^{-1}$ with $6 \mathrm{mg} \mathrm{L}^{-1}$ SDBS treatment. No living Poterioochromonas sp. were observed microscopically when the SDBS concentration was further increased to $8 \mathrm{mg} \mathrm{L}^{-1}$, which we considered as the complete control of Poterioochromonas sp. contamination.

The decreasing trend in Poterioochromonas sp. density with increasing surfactant concentration was found for all five tested surfactants, suggesting that they affect the predator Poterioochromonas sp. similarly. However, for each surfactant, the minimal effective concentrations to completely control the contamination were different. SDBS and AEO-7 were the most powerful reagents, eliminating Poterioochromonas sp. completely 

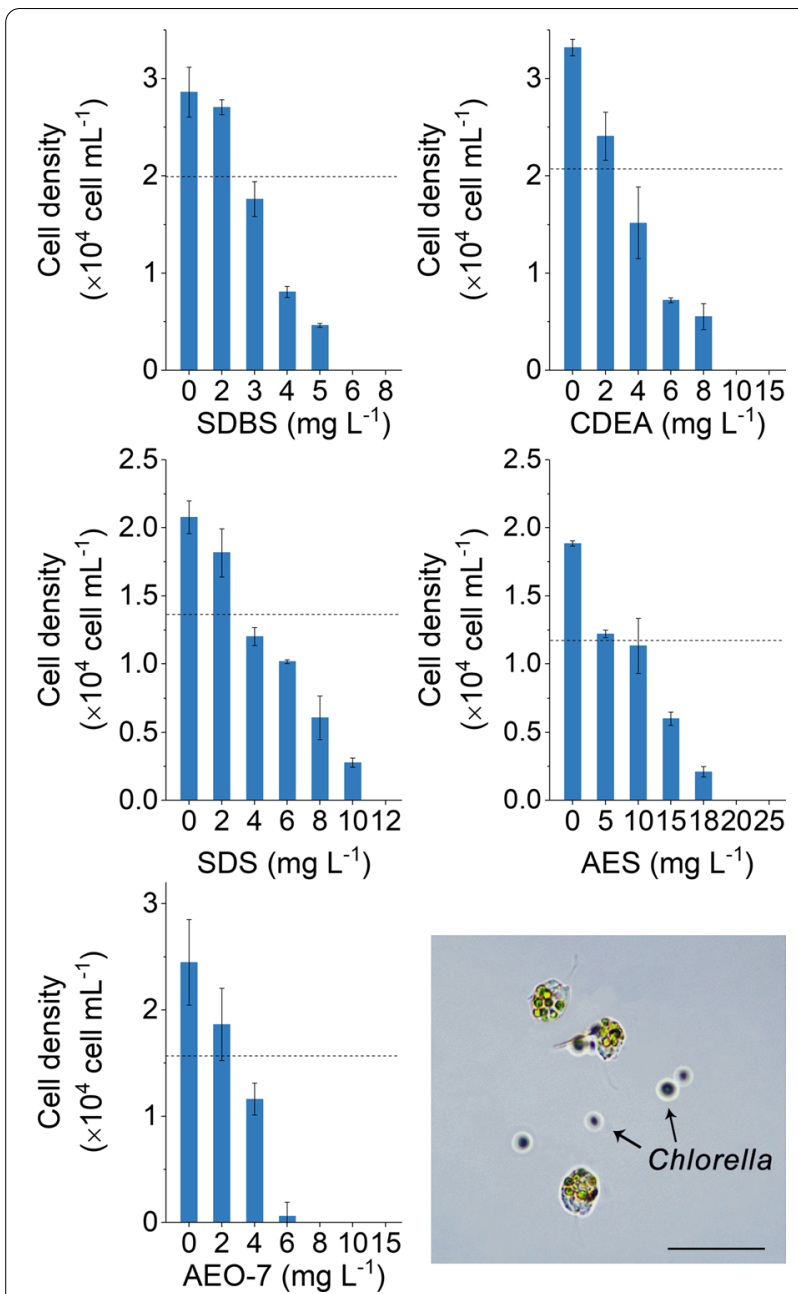

Fig. 1 Toxic effects of five surfactants on Poterioochromonas sp. growth. Dash lines represent cell densities at the beginning of the experiment. Columns indicate cell densities at $24 \mathrm{~h}$ after surfactant addition. Error bars represent the standard deviations of 3 replicates. Microscopic image of Poterioochromonas sp. with Chlorella cells inside their body is shown at the bottom-right corner. Scale bar $=30 \mu \mathrm{m}$

at concentrations not lower than $8 \mathrm{mg} \mathrm{L}^{-1}$. Second, the efficacies of CDEA and SDS on Poterioochromonas sp. were similar and their minimal effective concentrations were 10 and $12 \mathrm{mg} \mathrm{L}^{-1}$, respectively. AES showed weak efficacy on controlling of Poterioochromonas sp., with a minimal effective concentration of $20 \mathrm{mg} \mathrm{L}^{-1}$.

Toxic effects of the five surfactants on Hemiurosomoida sp. were also observed (Fig. 2). The viability of Hemiurosomoida sp. was shown by an increased in cell densities, which were more than $40 \%$ higher in comparison to the initial density in the culture without surfactant supplementation. Hemiurosomoida sp. densities decreased significantly after surfactants addition. Taking the SDBS treatment as an example, almost $60 \%$ decrease in the

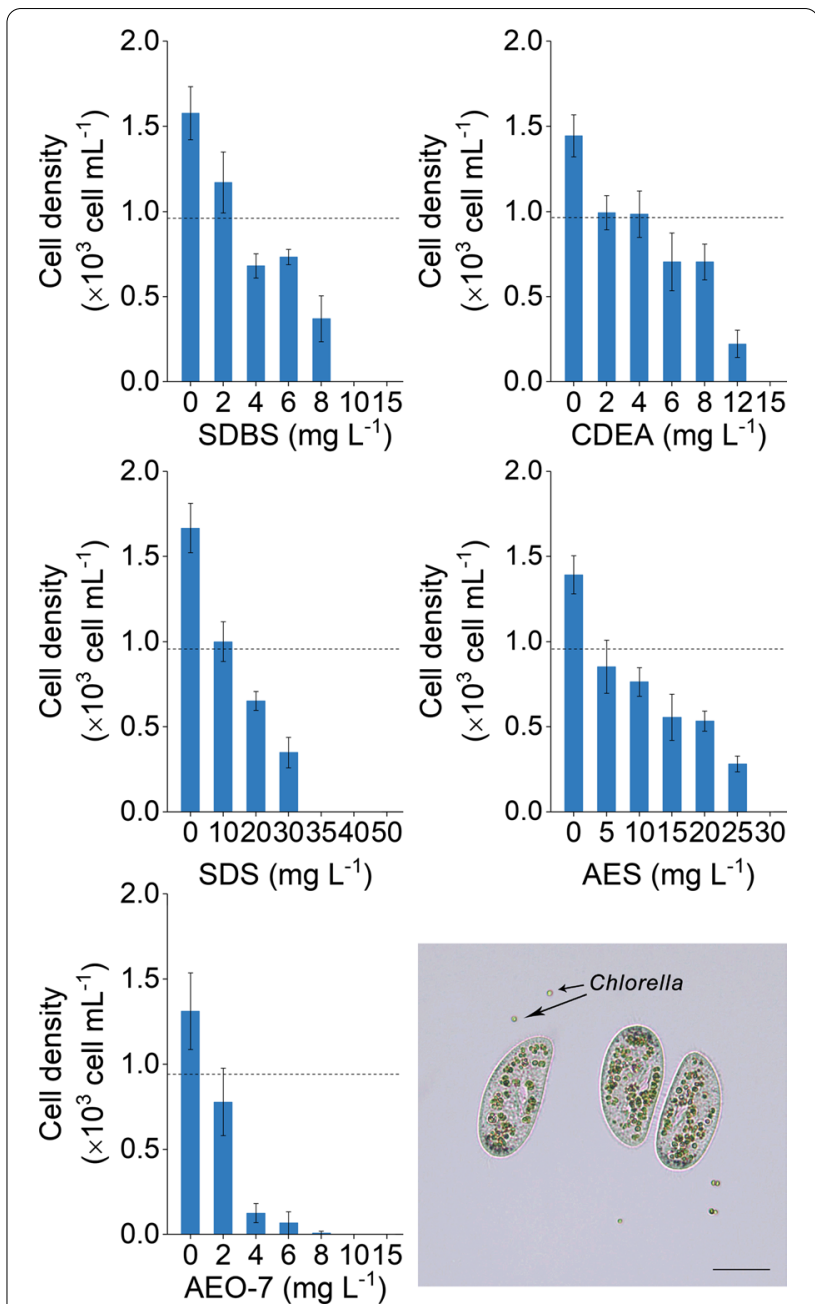

Fig. 2 Toxic effects of five surfactants on Hemiurosomoida sp. growth. Dash lines represent cell densities at the beginning of the experiment. Columns indicate cell densities at $24 \mathrm{~h}$ after surfactant addition. Error bars represent the standard deviations of 3 replicates. Microscopic image of Hemiurosomoida sp. with Chlorella cells inside their body is shown at the bottom-right corner. Scale bar $=60 \mu \mathrm{m}$

Hemiurosomoida sp. density, from $1.6 \times 10^{3}$ to 680 cells $\mathrm{mL}^{-1}$, was obtained when $4 \mathrm{mg} \mathrm{L}^{-1}$ SDBS was supplemented into the culture. A further increase in the SDBS concentration $\left(10 \mathrm{mg} \mathrm{L}^{-1}\right)$ led to the complete elimination of Hemiurosomoida sp. and no living cells were observed under the microscope. The general trends of decreasing cell densities with increasing surfactant concentrations were also detected for the five surfactants. However, the efficacies against Hemiurosomoida sp. were not the same as that for Poterioochromonas sp. The most powerful one was AEO-7, which eliminated Hemiurosomoida sp. at a concentration of $8 \mathrm{mg} \mathrm{L}^{-1}$. The next ones were SDBS and CDEA, the minimal effective concentrations of which were 10 and $15 \mathrm{mg} \mathrm{L}^{-1}$, respectively. The 
complete elimination of Hemiurosomoida sp. by AES was only obtained at $30 \mathrm{mg} \mathrm{L}^{-1}$. A substantial difference was observed in SDS, which had a minimal effective concentration of $12 \mathrm{mg} \mathrm{L}^{-1}$ for Poterioochromonas sp., but at least $35 \mathrm{mg} \mathrm{L}^{-1}$ SDS was needed to completely eliminate Hemiurosomoida sp.

\section{Effects of the five surfactants on Chlorella growth}

Chlorella pyrenoidosa XQ-20044 was cultured under different concentrations of the five surfactants to evaluate the surfactant effects on cell growth, photosynthetic activity, and viability. Data of the SDBS exposure experiment are shown in Fig. 3 as an example; other data concerning CDEA, SDS, AES, and AEO-7 are provided in Additional file 1.
The time courses of the Chlorella biomass DW showed no significant difference $(P>0.05)$ when the SDBS concentration was less than $20 \mathrm{mg} \mathrm{L}^{-1}$ (Fig. 3a). The biomass DW of the culture having no SDBS supplementation reached $0.72 \mathrm{~g} \mathrm{~L}^{-1}$ on day 3 , with an average growth rate of $0.84 \mathrm{~d}^{-1}$. Smaller but insignificant $(P>0.05)$ biomass DW $\left(0.67 \mathrm{~g} \mathrm{~L}^{-1}\right)$ and growth rate $\left(0.82 \mathrm{~d}^{-1}\right)$ were obtained in the culture with $20 \mathrm{mg} \mathrm{L}^{-1}$ SDBS supplementation. However, the biomass DW was only $0.41 \mathrm{~g}$ $\mathrm{L}^{-1}$ with a significantly decreased $(P<0.05)$ growth rate of $0.66 \mathrm{~d}^{-1}$ when the SDBS concentration was further increased to $40 \mathrm{mg} \mathrm{L}^{-1}$.

The photosynthetic activity of Chlorella (Fig. 3b) showed that in comparison to the SDBS-free culture, the changes in the photochemical yield of Chlorella
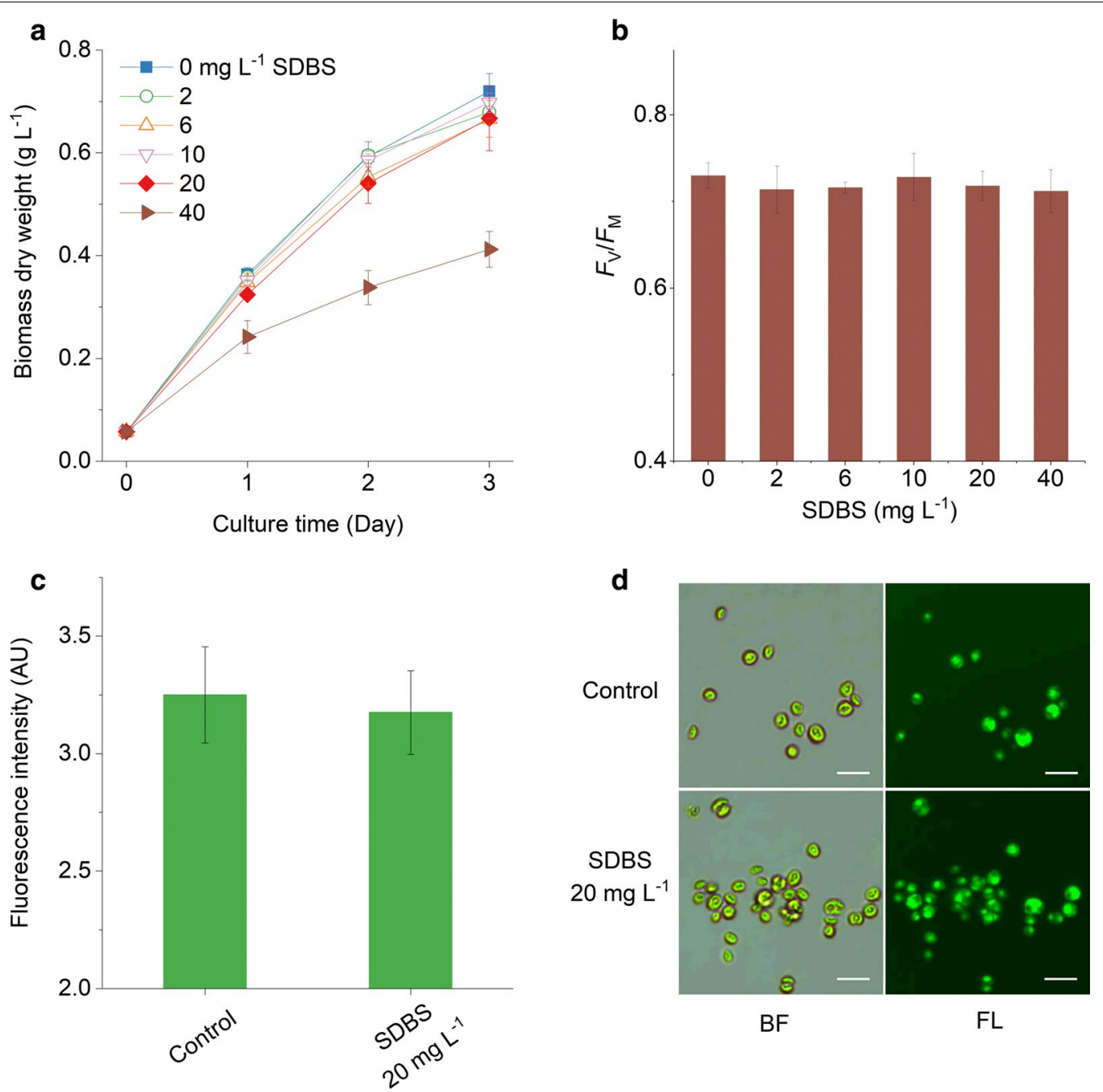

Fig. 3 Effects of sodium dodecyl benzene sulfonate (SDBS) on Chlorella pyrenoidosa XQ-20044. a Cell growth, b photosynthetic activity, c FDA staining fluorescence intensities, and $\mathbf{d}$ bright field (BF) and fluorescence (FL) image of the cells. Scale bars $=10 \mu \mathrm{m}$. Error bars represent the standard deviations of 3 replicates 
cells were very small after 3 days of exposure to $20 \mathrm{mg}$ $\mathrm{L}^{-1}$ SDBS. The ratio between variable fluorescence and maximum fluorescence $\left(F_{\mathrm{V}} / F_{\mathrm{M}}\right)$ of Chlorella was 0.72 in the SDBS-treated $\left(20 \mathrm{mg} \mathrm{L}^{-1}\right)$ culture in the present study. This value fell into the general $F_{\mathrm{V}} / F_{\mathrm{M}}$ range of dark-adapted green microalgae, suggesting that the photosynthetic activity of C. pyrenoidosa XQ-20044 was not influenced by SDBS at concentrations lower than $20 \mathrm{mg}$ $\mathrm{L}^{-1}$.

FDA staining (Fig. 3c, d) clearly showed membrane integrity and viability of the Chlorella cells, with similar fluorescein fluorescence intensities in both the SDBStreated $\left(20 \mathrm{mg} \mathrm{L}^{-1}\right)$ and the contrast culture. All the above results suggested that Chlorella biomass yield may be reduced due to over exposure to SDBS, but the influences of SDBS was negligible at a concentration not higher than $20 \mathrm{mg} \mathrm{L}^{-1}$.

\section{Application of sodium dodecyl benzene sulfonate (SDBS) as a pesticide to control flagellates and ciliates grazing on Chlorella in raceway pond}

The above results demonstrate that SDBS and AEO-7 were powerful surfactants for controlling Poterioochromonas and Hemiurosomoida contaminants while had little effect on Chlorella growth. Considering that SDBS is more easily degraded in natural environment and is less toxic to aquatic organisms than AEO-7 [23], SDBS was further tested outdoors to validate the laboratory data. According to our observation, naturally occurring contaminations of Poterioochromonas sp. or Hemiurosomoida sp. can be observed generally on days $2-4$ of a newly inoculated Chlorella culture in an outdoor raceway pond (unpublished results). This trend was successfully mimicked by the addition of Poterioochromonas sp. or Hemiurosomoida sp. "seeds" into the Chlorella culture ponds (Fig. 4). 18S rDNA-based metagenomic data for identification of the contaminating species can be seen in Additional file 2. The predator densities increased continuously for 3-4 days after inoculation. For example, Hemiurosomoida $\mathrm{sp}$. increased from $1.0 \times 10^{5}$ cells $\mathrm{L}^{-1}$ on the 4th day to $1.4 \times 10^{6}$ cells $\mathrm{L}^{-1}$ on the 7 th day. At this time the cultures were treated with $10 \mathrm{mg} \mathrm{L}^{-1}$ SDBS, and contaminations in other parallel cultures were not treated and allowed to develop.

As shown in Fig. 4, cell densities of the predators Poterioochromonas sp. and Hemiurosomoida sp. increased consistently for 3 or 4 days. The target microalgae $C$. pyrenoidosa XQ-20044 also showed a quick increase in cell density (indicated by Chl $a$ content) during this period because the predator populations were not large enough to have a significant grazing effect on Chlorella. The increase in predator densities continued thereafter in the cultivations without SDBS addition. When the densities of Poterioochromonas sp. and Hemiurosomoida sp. reached approximately $3.6 \times 10^{7}$ and $6.4 \times 10^{5}$ cells $\mathrm{L}^{-1}$, respectively, the Chlorella density decreased due to predation. By comparison, almost all the Poterioochromonas sp. and Hemiurosomoida sp. cells disintegrated and disappeared in one day in the cultivations after SDBS addition $\left(10 \mathrm{mg} \mathrm{L}^{-1}\right)$ on the 6th day and 7th day, respectively, with the Chlorella growth kept as normal.

Overall, the final Chlorella biomass concentration reached $0.6 \mathrm{~g} \mathrm{~L}^{-1}$ after a 12-day cultivation applying SDBS as pesticide. It was only $0.26 \mathrm{~g} \mathrm{~L}^{-1}$ if the
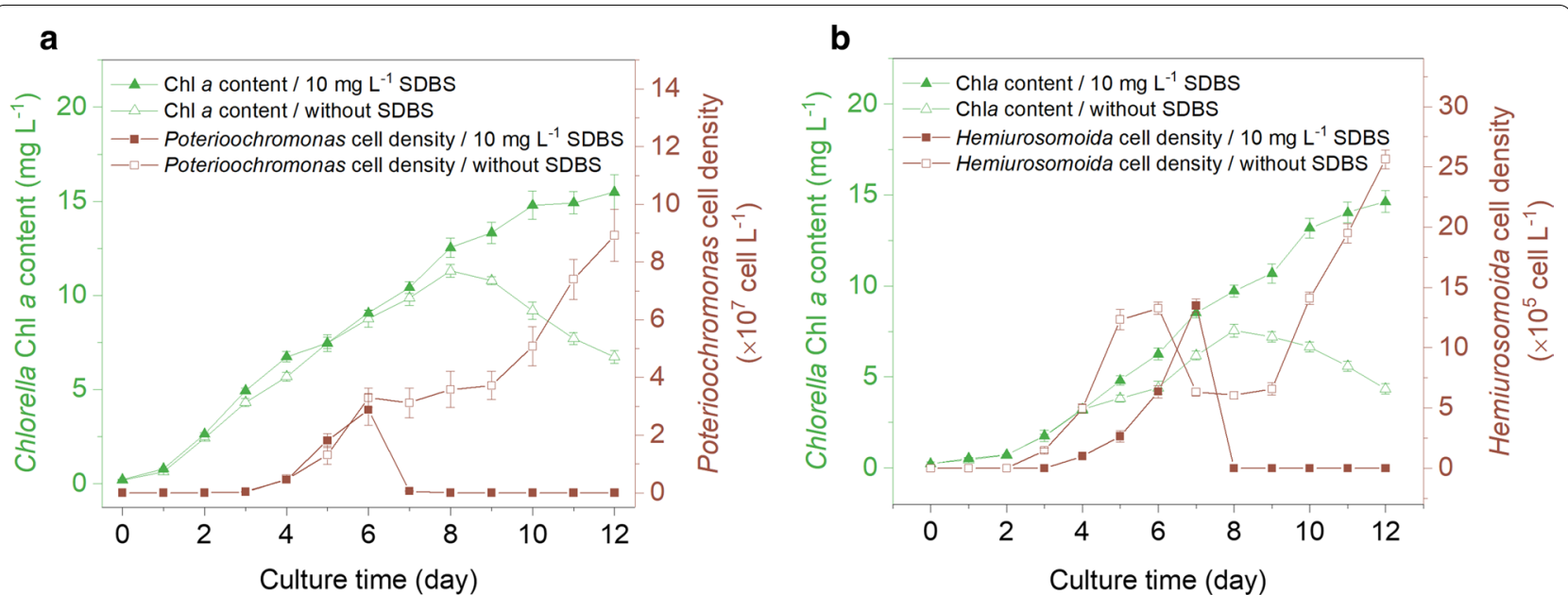

Fig. 4 Changes in cell density of Chlorella and inoculated contaminating organisms in 5- $\mathrm{m}^{2}$ raceway ponds. The contaminating organisms were Poterioochromonas sp. (a) and Hemiurosomoida sp. (b), with open symbols indicating the cultivation without SDBS treatment, and closed symbols indicating the cultivation treated with $10 \mathrm{mg} \mathrm{L}^{-1}$ SDBS on the 6 th day (a) and the 7th day (b). Error bars represent the standard deviations of 3 replicate measurements 


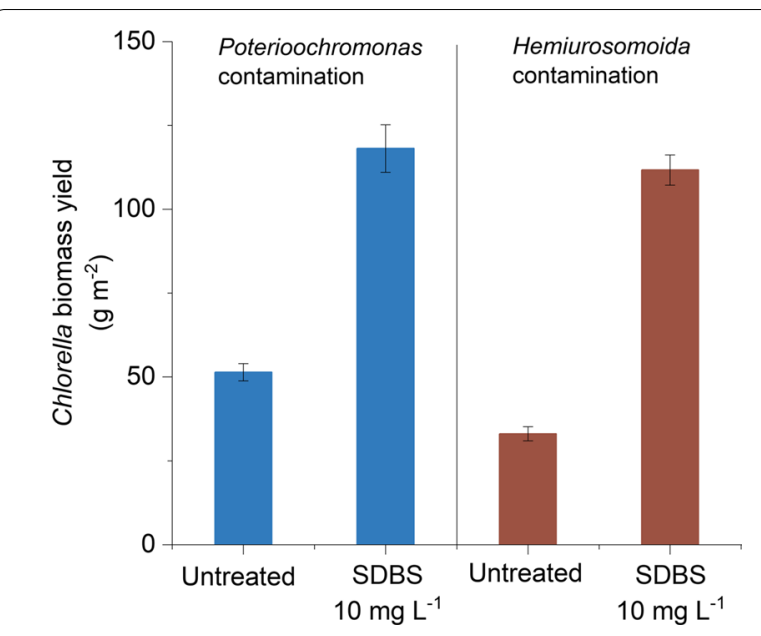

Fig. 5 Comparison of biomass yields between the contaminated cultivations with and without SDBS treatment. Error bars represent the standard deviations of 3 replicate measurements
Poterioochromonas contamination was not controlled and $0.17 \mathrm{~g} \mathrm{~L}^{-1}$ if the Hemiurosomoida contamination was not controlled (Fig. 5). These data suggest that by applying $10 \mathrm{mg} \mathrm{L}^{-1} \mathrm{SDBS}$ as a pesticide to control Poterioochromonas sp. or Hemiurosomoida sp. contamination, the reduction in Chlorella biomass yield, which was estimated to be greater than $60 \%$ owing to predation, can be avoided. Actually, economic loss caused by biological contamination was much bigger than expected because the residual Chlorella biomass could only be used as low-quality raw materials when no effective steps were taken to manage the contaminations. The working concentration of SDBS $\left(10 \mathrm{mg} \mathrm{L}^{-1}\right)$ was slightly higher than the minimal effective concentration to eliminate Poterioochromonas sp. in the laboratory. This was to simplify the application that using one uniform concentration to control both Poterioochromonas and Hemiurosomoida contaminations.

SDBS pesticide was also applied in 20 and $200 \mathrm{~m}^{2}$ cascade cultures of Chlorella at October 2019 (Fig. 6). The cascade cultures were initiated in a $20-\mathrm{m}^{2}$ raceway pond. After 10 days of cultivation the culture suspension was used as seed for a larger culture in a $200-\mathrm{m}^{2}$ raceway pond. Two rounds of contamination naturally occurred during the process, both of which were Poterioochromonas sp. contaminations. The first round of Poterioochromonas contamination was observed early on the 2 nd day in the $20-\mathrm{m}^{2}$ pond. The cell density of Poterioochromonas increased gradually from $7.6 \times 10^{4}$ to $1.1 \times 10^{6}$ cells $\mathrm{L}^{-1}$ on the $3 \mathrm{rd}$ day, and then drastically increased with densities on the 4 th and 5 th days reaching $8.1 \times 10^{6}$ and $2.8 \times 10^{7}$ cells $\mathrm{L}^{-1}$, respectively. During this time, the Chlorella density was not significantly

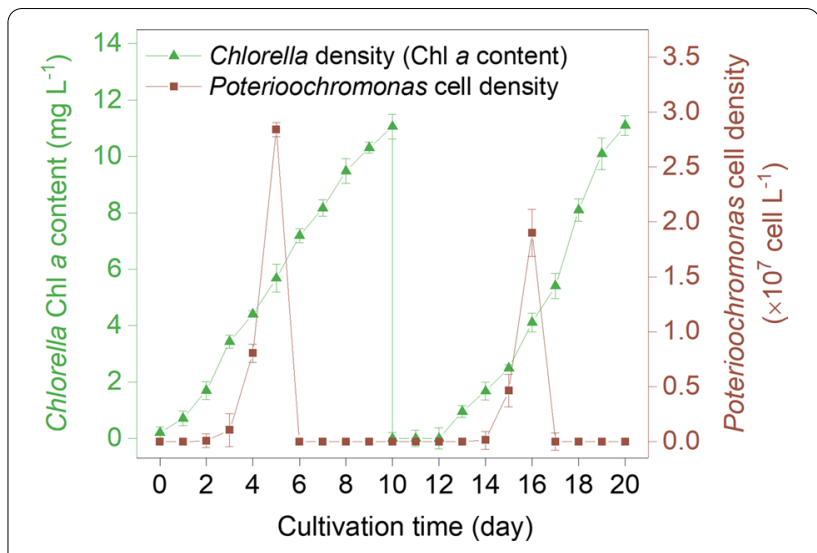

Fig. 6 Cascade cultivations of Chlorella in raceway ponds and contamination control using SDBS. The first 10 days of cultivation was conducted in a $20-\mathrm{m}^{2}$ pond and the next 10 days was in a $200-\mathrm{m}^{2}$ raceway pond. Both observed contaminants were Poterioochromonas sp. and $10 \mathrm{mg} \mathrm{L}^{-1}$ SDBS was added on the 5th and 16th day. Error bars represent the standard deviations of 3 replicate measurements

influenced because the predator density was relatively low. SDBS addition $\left(10 \mathrm{mg} \mathrm{L}^{-1}\right)$ on the 5 th day resulted in a sharp decrease in Poterioochromonas sp. density and the predator was not observed over the following days. The algal biomass increased continuously after addition of the SDBS pesticide. A cell density of $11.1 \mathrm{mg} \mathrm{Chl}$ $a \mathrm{~L}^{-1}\left(0.42 \mathrm{~g} \mathrm{DW} \mathrm{L}^{-1}\right.$, alternatively $\left.8.4 \mathrm{~g} \mathrm{~m}^{-2} \mathrm{~d}^{-1}\right)$ was observed on the 10th day. The biomass yield of Chlorella was comparable to those previously reported [24, 25]. On the 10th day, the culture was scaled up into a 200$\mathrm{m}^{2}$ raceway pond and 4 days later the second round of Poterioochromonas contamination was observed. Development of the second round of contamination was very similar to the previous one observed in the $20-\mathrm{m}^{2}$ pond. SDBS pesticide $\left(10 \mathrm{mg} \mathrm{L}^{-1}\right)$ successfully eliminated Poterioochromonas sp. once again, without damaging Chlorella growth.

\section{Discussion}

Surfactants as novel pesticide for controlling biological contamination in Chlorella culture

In the present study surfactants were used as pesticide to control ciliate and flagellate contaminations in Chlorella culture. Among the selected five surfactants, SDBS, SDS, and AEO-7 met the basic requirements of a pesticide for the control of Poterioochromonas sp. and Hemiurosomoida sp. in Chlorella culture. First, the complete control (elimination) of the two predators could be achieved by the addition of any one of the tested surfactants at a proper concentration (Figs. 1 and 2). Second, the surfactants SDBS, SDS, and AEO-7, which eliminated the two predators at the minimal effective concentrations, 
had little effects on Chlorella growth. Particularly, the minimal effective concentrations of SDBS for the complete elimination of Poterioochromonas sp. and Hemiurosomoida sp. were as low as 8 and $10 \mathrm{mg} \mathrm{L}^{-1}$, respectively. However, SDBS concentrations as high as $20 \mathrm{mg} \mathrm{L}^{-1}$ had no effect on photosynthetic activity, cell membrane integrity, and biomass accumulation of C. pyrenoidosa XQ-20044 (Fig. 3).

\section{Possible mechanisms of SDBS pesticide for controlling bio-contaminations}

Previous studies regarding the aquatic toxicity of anionic surfactants $[15,16,26,27]$ showed that green algae were more tolerant to anionic surfactant (such as SDBS) exposure compared to invertebrates including daphnia, ciliates, flagellates, and bacteria. These results were consistent with those of the present study. Such differential tolerance between Chlorella and the two predators provide evidence that these surfactants can be used as pesticides to control contamination in Chlorella mass cultures.

One remaining question is why did the surfactants only eliminate predators such as Poterioochromonas sp. and Hemiurosomoida sp. rather than Chlorella? Microscopical observation at $24 \mathrm{~h}$ after the addition of surfactants showed that the predators decreased in numbers or even disappeared from the Chlorella culture. In fact, these changes occurred in less than $10 \mathrm{~min}$ after the addition of surfactants. Continuous microscopic monitoring revealed that the predator cells, whether it was Poterioochromonas sp. or Hemiurosomoida sp., disintegrated shortly once the SDBS concentration got close to the minimal effective concentration (Additional file 3). However, the free-living Chlorella cells that were not swallowed retained their morphological and physiological integrity (Fig. 3).

As mentioned before, cell membranes are the primary target for the toxicological effects of surfactants on cell $[18,19]$. Poterioochromonas and Hemiurosomoida are unicellular organisms that lack a rigid or resistant structure (composed of insoluble non-hydrolysable biopolymers) outside the plasma membrane [20-22]. These cells were so sensitive that the lipid bilayers were disrupted immediately when enough surfactants were available in the medium.

One of the cell structures that differs Chlorella from the two predators (Poterioochromonas sp. and Hemiurosomoida sp.) is its cell wall. Numerous species of green microalgae including C. pyrenoidosa have a two-layer cell wall with a classical polysaccharidic layer that is proximal to the cytoplasmic membrane and a thin outer layer $[28,29]$. The outer layers are often trilaminar organized (termed as the trilaminar sheath, TLS) and are composed of insoluble non-hydrolysable biopolymers exhibiting an unusually high resistance to non-oxidative chemical degradation [30, 31]. In a study concerning bio-toxicity of environmental chemicals, Gwenael Corre et al. [28] found that the presence of a TLS in C. emersonii was associated with a very high resistance to anionic (DBS) and nonionic (TX-100) detergents at all growth stages. We consider this is also the reason why the photosynthetic capacity and viability of $C$. pyrenoidosa were not significantly affected by $20 \mathrm{mg} \mathrm{L}^{-1}$ SDBS in the present study. The TLS of $C$. pyrenoidosa may have worked as a protective structure against SDBS.

\section{Applications of SDBS pesticide in Chlorella mass culture}

SDBS is one of the most commonly used anionic surfactants for cleaning application [32], and it is easy to manufacture, store, transport, and handle [32], which facilitate its application in microalgae production. The current price of SDBS is $\$ 1.4$ per kilogram in China. In other words, material cost is only 1.4 U.S. dollar for applying SDBS pesticide to a $100 \mathrm{~m}^{3}$ Chlorella cultivation. It is very cheap in comparison to other methods [33].

SDBS is generally regarded as a biodegradable surfactant and its degradation rate may be as high as $97-99 \%$ under aerobic conditions [34]. We harvested the SDBStreated Chlorella in other study [33] to find if there was residual SDBS in the harvested Chlorella biomass, and no SDBS was detected. Thus, the environmental impact of this method is slight. However, more investigations are still needed. Our previous studies have also shown that the surfactants such as SDBS are unable to induce changes in algal lipid synthesis $[35,36]$. Therefore, it will be very cheap, convenient, and safe to use SDBS as a new pesticide in microalgal mass cultivation, especially for biofuel production. Avoiding target biomass reduction is a necessary principle for biological contamination control in microalgal cultivation. Predator reproduction and Chlorella biomass loss are both becoming faster and bigger with the extension of time for a contaminated Chlorella cultivation. So, early detection and treatment are crucial for minimizing algal biomass reduction. From this point of view, $10 \mathrm{mg} \mathrm{L}^{-1}$ SDBS should be added as soon as predators are observed microscopically to prevent further reproduction of predators. Since cell densities of the predator Poterioochromonas and Hemiurosomoida are relatively low at this time, $10 \mathrm{mg} \mathrm{L}^{-1}$ SDBS would be adequate for completely eliminating the contaminants.

The tolerable SDBS concentration for C. pyrenoidosa is $20 \mathrm{mg} \mathrm{L}^{-1}$, which is at least two times that of the minimal effective concentration for eliminating the predators Poterioochromonas sp. and Hemiurosomoida sp. Such a difference is very helpful for outdoor application. Even 
the SDBS pesticide is required once again in a short time, the Chlorella will not be affected negatively. On the other hand, predator cells are disintegrated in a short time after SDBS addition, thus protective time of the pesticide will depend on how fast SDBS is degraded. Further studies should concern degradation dynamics of SDBS in microalgal culture and how environmental factors affect the degradation process.

\section{Conclusions}

All five selected surfactants were effective for eliminating Poterioochromonas sp. and Hemiurosomoida sp. contamination in the laboratory. Further studies indicated SDBS $\left(10 \mathrm{mg} \mathrm{L}^{-1}\right)$ is an efficient pesticide to control the contaminations without damaging Chlorella. One of the principles for SDBS pesticide application is early detection and treatment of contaminations. The surfactant SDBS directly acts on the unprotected plasma membrane of the predators; therefore, the efficacy of SDBS as a pesticide may be universal. The authors expect a broad spectrum of anti-biocontaminations to be developed using the method outlined in the present study.

\section{Methods}

Chemicals used for the control of biological contaminants Five surfactants, specifically sodium dodecyl benzene sulfonate (SDBS, CAS NO. 25155-30-0), coconut diethanolamide (CDEA, CAS NO. 68603-42-9), sodium dodecyl sulfate (SDS, CAS NO. 151-21-3), fatty alcohol polyoxyethylene ether (AEO-7, C12, CAS NO. 6813139-5), and alcohol ethoxysulphate (AES, C12, CAS NO. 68891-38-3), were tested for their effects on microalgae and biological contaminants.

\section{Chlorella strain and test for its growth under surfactants exposure}

Chlorella pyrenoidosa XQ-20044 was used in the present study. It was provided by the Algae Culture Collection of Wuhan Botanical Garden, Chinese Academy of Sciences. The algal seed were grown autotrophically in shaking flasks under light intensity of $50 \mu \mathrm{mol} \mathrm{m} \mathrm{m}^{-2} \mathrm{~s}^{-1}$ for $14 \mathrm{~h}$ per day and constant temperature $\left(25^{\circ} \mathrm{C}\right)$.

To study the effects of the SDBS surfactant on Chlorella growth, the seed cultures were inoculated into a bubbled column photobioreactor (PBR) at an initial density of $\mathrm{OD}_{540}=0.3$ (approximately $0.05 \mathrm{~g} \mathrm{~L}^{-1}$ dry weight). Light intensity at the surface of the column PBR was set at $200 \mu \mathrm{mol} \mathrm{m} \mathrm{m}^{-2} \mathrm{~s}^{-1}$ with a light/dark cycle of $14 / 10 \mathrm{~h}$. A thermostatic water bath was used to maintain constant culture temperature of $30{ }^{\circ} \mathrm{C}$. The culture suspension was agitated with $200 \mathrm{~mL} \mathrm{~min}^{-1}$ air enriched with $1 \% \mathrm{CO}_{2}$ (v/v). The basal growth medium was BG-11. Concentrated SDBS solutions were added to the culture columns immediately after inoculation to reach final SDBS concentrations of $0,2,6,10,20$, and $40 \mathrm{mg} \mathrm{L}^{-1}$. Each cultivation was run in triplicate and proceeded for 3 days. The biomass dry weight (DW) and photosynthesis activity were monitored every day during cultivation. Fluorescein diacetate (FDA) dye was used to indicate the cell viability of Chlorella pyrenoidosa XQ-20044. Effects of the other four surfactants were evaluated by comparing DWs of the culture under the same conditions.

\section{Predator isolation and successive transfer cultures}

The predators, namely Poterioochromonas sp. and Hemiurosomoida sp., were frequently observed and caused biomass loss during our test cultivations of C. pyrenoidosa XQ-20044 in open raceway ponds from 2011 to 2019. We isolated Poterioochromonas sp. and Hemiurosomoida sp. cells and fed them C. pyrenoidosa XQ-20044 cells. After repeatedly re-isolation and feeding, steady cultures of the two predators were established. For maintenance, the cultures were kept at room temperature $\left(25{ }^{\circ} \mathrm{C}\right)$ with manual shaking (twice per day). Diluted C. pyrenoidosa XQ-20044 suspension (approximately $\mathrm{OD}_{540}=0.1$ ) was fed to the Poterioochromonas sp. or Hemiurosomoida sp. cultures in a mixed ratio of $1: 1(\mathrm{v} / \mathrm{v})$ every 2-3 days. By this way, successive transfer cultures of Poterioochromonas sp. and Hemiurosomoida sp. were established, which greatly facilitated the experiments.

\section{Control of Poterioochromonas sp. and Hemiurosomoida sp. using surfactants}

Based on the successive transfer cultures of the two predators, the effects of SDBS, CDEA, SDS, AEO-7, and AES on Poterioochromonas sp. and Hemiurosomoida sp. were studied. Immediately after feeding with Chlorella, the cell densities of Poterioochromonas sp. and Hemiurosomoida sp. were counted and recorded. Generally, by feeding $1 \mathrm{~L}$ of the grazer culture with $1 \mathrm{~L}$ diluted Chlorella suspension every 2 days, the cell densities of Poterioochromonas sp. and Hemiurosomoida sp. reached at least $10^{4}$ cells $\mathrm{mL}^{-1}$ and $8 \times 10^{2}$ cells $\mathrm{mL}^{-1}$, respectively, which ensured fast growth during the following days if no surfactant was added. These predator cultures were transferred into small flasks ( $80 \mathrm{~mL}$ working volume) and different volumes of the concentrated surfactant solutions were added to reach the desired concentrations. The flasks were kept at room temperature $\left(25^{\circ} \mathrm{C}\right)$ with low light and occasional shaking. The experiments were performed in triplicate. A control culture that without surfactant addition was included in each experiment to show predator viability and effectiveness of using the successive transfer culture. Morphological changes during the first hour were observed using an optical microscope and recorded with a digital camera. After 24 h of surfactant exposure, 
the cell densities of the live Poterioochromonas sp. and Hemiurosomoida sp. were counted.

\section{Field test of sodium dodecyl benzene sulfonate (SDBS) as a pesticide to control predatory flagellate and ciliate contaminations}

SDBS was used as a pesticide to control Poterioochromonas sp. and Hemiurosomoida sp. in Chlorella mass cultivation. The field test was conducted at the microalgal mass culture test station at Yunnan province, China $\left(26^{\circ} 29^{\prime} 29.6^{\prime \prime} \mathrm{N} ; 100^{\circ} 40^{\prime} 56.12^{\prime \prime} \mathrm{E}\right)$. Detailed information about the raceway ponds and general cultivation parameters can be seen in our previous study [37]. Chlorella pyrenoidosa XQ-20044 was firstly cultivated in greenhouse-covered raceway ponds $\left(5 \mathrm{~m}^{2}, 1000 \mathrm{~L}\right)$. Then, approximately $2 \mathrm{~L}$ of the Poterioochromonas sp./Hemiurosomoida sp. culture suspension was added empirically into each pond on the 2 nd or 3 rd day. The grazer cultures acted as seeds to bring about Poterioochromonas sp./Hemiurosomoida sp. contamination, which was validated later by microbial community analysis using metagenomics data. $1-2$ days after the addition, the predators increased in density and could be easily observed under the microscope and counted using a counting chamber. After several days of cultivation, the predator density had a marked increase, and then $10 \mathrm{mg}$ $\mathrm{L}^{-1}$ SDBS was added to the ponds. For the control experiments, the development of the two predators was not interfered with by any extra operation. The experiments were conducted in parallel. Chl $a$ content and predator density were monitored every day to indicate Chlorella growth and predator development, respectively.

The SDBS pesticide was also applied in a $20-200 \mathrm{~m}^{2}$ cascade culture of Chlorella in October 2019. The cultivations were performed according to our previous study [37] and continued for 20 days. For the first 10 days, the cultivation was conducted in a greenhouse-covered $20-\mathrm{m}^{2}$ raceway pond (4000 $\mathrm{L}$ cultural volume) and then the culture suspension was transferred to a $200-\mathrm{m}^{2}$ open raceway pond (40,000 $\mathrm{L}$ cultural volume) to inoculate a new cultivation. The cascade culture was microscopically monitored twice a day and two rounds of naturally occurring bio-contamination were observed. The SDBS pesticide $\left(10 \mathrm{mg} \mathrm{L}^{-1}\right)$ was used to control these contaminations.

\section{Measurements}

Biomass dry weight (DW) and Chlorophyll $a(\mathrm{Chl} a)$ content were measured according to previous study [38] to evaluate Chlorella growth.. An equation (DW g $\left.\mathrm{L}^{-1}=38.14 \times \mathrm{Chl} a \mathrm{mg} \mathrm{mL}^{-1}, R^{2}=0.9979\right)$ was estimated from an uncontaminated parallel culture and used to calculate Chlorella DW for those samples that had contamination during the field test.

The photosynthetic status of Chlorella was evaluated by measuring the chlorophyll fluorescence parameter $\left(F_{\mathrm{V}} / F_{\mathrm{M}}\right)$ using a PAM 2500 fluorometer according to Xie et al.s method [39]. Fluorescein diacetate (FDA) staining was used to determine Chlorella viability (cell membrane integrity) according to the methodology described by Serra-Maia et al. [40]. Pictures were also taken on a Leica DMi8 C microsystem.

Cell densities of Poterioochromonas sp. and Hemiurosomoida sp. were counted with two types of plankton counting chamber $(0.1 \mathrm{~mL}, 400$ counting squares and $0.1 \mathrm{~mL}, 100$ counting squares) after fixing with Lugol's solution. Only $1 \mu \mathrm{L}$ of Lugol's solution (10\%) was used for each $10 \mathrm{~mL}$ of sample to inhibit predator swimming but avoid cell disruption. At least three independent countings were conducted for each sample. As surfactants induced cell disintegration, all intact cells observed under microscope were recognized as live cell. To study the morphological changes of predator cells exposure to surfactant, the cells were continuously monitored under microscope, and a small device was used to assist video recording. Description of the device and the recorded videos can be seen in Additional file 3. The outdoor samples were also subjected to metagenomic sequencing to evaluate whether the microbial community was consistent with that expected.

All the above analytical experiments were performed in triplicate and the results were analyzed for variance using SAS 9.13 at a significance level of $\alpha=0.05$. Tukey's multiple comparison tests were done where applicable.

\section{Supplementary Information}

The online version contains supplementary material available at https://doi. org/10.1186/s13068-021-01873-6.

Additional file 1. Effects of surfactants (CDEA, SDS, AES, AEO-7) on growth of the alga Chlorella pyrenoidosa XQ-20044.

Additional file 2. Taxonomic classification of organisms in Chlorella cultures ( $5 \mathrm{~m}^{2}$ raceway pond) using metagenomics data at the 3rd day after inoculation with Poterioochromonas or Hemiurosomoida.

Additional file 3. Videos showing disintegration of Poterioochromonas and Hemiurosomoida cells exposure to SDBS pesticide (10 mg /L) and schematic diagram of the device used to assist video recording.

Acknowledgements

The authors thank Yunnan Green A Biological Engineering Co., Ltd for providing help in the open pond cultures.

Authors' contributions

XW performed indoor and outdoor cultivations and examinations, analyzed and interpreted the data, and draft the manuscript. AZ, YD, XZ participated in the outdoor cultivation and examination. $\mathrm{LL}, \mathrm{YH}, \mathrm{KW}$, and $Y Y$ participated in the laboratory investigation and data visualization. YG, YD and YL designed 
the experiment and critically revised the manuscript. All authors read and approved the final manuscript.

\section{Funding}

This work was supported by the National Natural Science Foundation of China (No. 31702367) and China Petrochemical Corporation (No. 210080, No. 215104)

\section{Availability of data and materials}

All data generated or analyzed during this study are included in this published article and its supplementary information files.

\section{Ethics approval and consent to participate}

Not applicable.

\section{Consent for publication}

Not applicable.

\section{Competing interests}

The authors declare that they have no competing interests.

\section{Author details}

1 Key Laboratory of Plant Germplasm Enhancement and Specialty Agriculture, Wuhan Botanical Garden, Chinese Academy of Sciences, Wuhan 430074, China. ${ }^{2}$ Center of Economic Botany, Core Botanical Gardens, Chinese Academy of Sciences, Wuhan 430074, China. ${ }^{3}$ University of Chinese Academy of Sciences, Beijing 100049, China.

Received: 10 September 2020 Accepted: 1 January 2021 Published online: 14 January 2021

\section{References}

1. Masojídek J, Torzillo G. Mass cultivation of freshwater microalgae. In: Jørgensen SE, Fath BD, editors. Encyclopedia of Ecology. California: Elsevier; 2008. p. 2226-35

2. Preisig HR, Andersen RA. Historical review of algal culturing techniques. In: Andersen RA, editor. Algal culturing techniques. California: Elsevier Academic Press; 2005. p. 1-12.

3. Liu J, Hu Q. Chlorella: industrial production of cell mass and chemicals. In: Richmond A, Hu Q, editors. Handbook of Microalgal Culture: Applied Phycology and Biotechnology. 2nd ed. Oxford: Blackwell Publishing; 2013. p. 327-38.

4. Ramanan R, Kannan K, Deshkar A, Yadav R, Chakrabarti T. Enhanced algal $\mathrm{CO} 2$ sequestration through calcite deposition by Chlorella sp. and Spirulina platensis in a mini-raceway pond. Bioresour Technol. 2009;101(8):2616-22.

5. Guccione A, Biondi N, Sampietro G, Rodolfi L, Bassi N, Tredici M. Chlorella for protein and biofuels: from strain selection to outdoor cultivation in a Green Wall Panel photobioreactor. Biotechnol Biofuels. 2014;7(1):84.

6. Wang H, Zhang W, Chen L, Wang J, Liu T. The contamination and control of biological pollutants in mass cultivation of microalgae. Bioresour Technol. 2013;128:745-50.

7. Lam TP, Lee TM, Chen CY, Chang JS. Strategies to control biological contaminants during microalgal cultivation in open ponds. Bioresour Technol. 2018;252:180-7.

8. Day JG, Gong Y, Hu Q. Microzooplanktonic grazers - a potentially devastating threat to the commercial success of microalgal mass culture. Algal Res. 2017;27:356-65.

9. Wang Y, Gong Y, Dai L, Sommerfeld M, Zhang C, Hu Q. Identification of harmful protozoa in outdoor cultivation of Chlorella and the use of ultrasonication to control contamination. Algal Res. 2018;31:298-310.

10. Ma M, Yuan D, He Y, Park M, Gong Y, Hu Q. Effective control of Poterioochromonas malhamensis in pilot-scale culture of Chlorella sorokiniana GT-1 by maintaining $\mathrm{CO}_{2}$-mediated low culture pH. Algal Res. 2017;26:436-44.

11. Ma M, Gong Y, Hu Q. Identification and feeding characteristics of the mixotrophic flagellate Poterioochromonas malhamensis, a microalgal predator isolated from outdoor massive Chlorella culture. Algal Res. 2018;29:142-53.
12. Moreno-Garrido I, Cañavate JP. Assessing chemical compounds for controlling predator ciliates in outdoor mass cultures of the green algae Dunaliella salina. Aquacult Eng. 2001;24(2):107-14.

13. Day JG. Grazers: the overlooked threat to the sustained production of future algal biofuels. Biofuels. 2013;4(5):459-61.

14. Karsa DR. What are Surfactants? In: Farn RJ, editor. Chemistry and technology of surfactants. Oxford: Blackwell Publishing; 2006. p. 1-23.

15. Könnecker G, Regelmann J, Belanger S, Gamon K, Sedlak R. Environmental properties and aquatic hazard assessment of anionic surfactants: physico-chemical, environmental fate and ecotoxicity properties. Ecotoxicol Environ Saf. 2011;74(6):1445-60.

16. Lechuga M, Fernández-Serrano M, Jurado E, Núñez-Olea J, Ríos F. Acute toxicity of anionic and non-ionic surfactants to aquatic organisms. Ecotoxicol Environ Saf. 2016;125:1-8.

17. Cserháti T, Forgács E, Oros G. Biological activity and environmental impact of anionic surfactants. Environ Int. 2002;28(5):337-48.

18. Woldringh CL. Lysis of the cell membrane of Escherichia coli $\mathrm{K} 12$ by ionic detergents. Biochim Biophys Acta. 1970;224(1):288-90.

19. Partearroyo MA, Ostolaza H, Goñi FM, Barberá-Guillem E. Surfactantinduced cell toxicity and cell lysis: A study using B16 melanoma cells. Biochem Pharmacol. 1990;40(6):1323-8.

20. Guo S, Song L. Observation on Poterioochromonas sp. (Chrysophyte). J Water Res Protect. 2010;2:592-6.

21. Shen YF, Wang JG. Protozoology. Beijing: Science Press; 1999. ((In Chinese)).

22. Dong J, Li L, Fan X, Ma H, Warren A. Two Urosoma species (Ciliophora, Hypotrichia): a multi-disciplinary approach provides new insights into their ultrastructure and systematics. Eur J Protistol. 2020;72:125661.

23. Versteeg J, Stanton T, Pence A, Cowan C. Effects of surfactants on the rotifer, Brachionus calyciflorus, in a chronic toxicity test and in the development of QSARs. Environ Toxicol Chem. 1997;16:1051-8.

24. De Bhowmick G, Subramanian G, Mishra S, Sen R. Raceway pond cultivation of a marine microalga of Indian origin for biomass and lipid production: a case study. Algal Res. 2014;6:201-9.

25. Matos ÂP, Ferreira WB, Morioka LRI, Moecke EHS, SantAnna ES. Cultivation of Chlorella vulgaris in medium supplemented with desalination concentrate grown in a pilot-scale open raceway. Braz J Chem Eng. 2018;35(4):1183-92.

26. Fendinger NJ, Versteeg DJ, Weeg E, Dyer S, Rapaport RA. Environmental behavior and fate of anionic surfactants. In: Baker LA, editor. Environmental chemistry of lakes and reservoirs. Washington: American Chemical Society; 1994. p. 527-57.

27. Jørgensen $E$, Christoffersen K. Short-term effects of linear alkylbenzene sulfonate on freshwater plankton studied under field conditions. Environ Toxicol Chem. 2000;19(4):904-11.

28. Corre G, Templier J, Largeau C, Rousseau B, Berkaloff C. Influence of cell wall composition on the resistance of two Chlorella species to detergents. J Phycol. 1996;32(4):584-90.

29. Northcote DH, Goulding KJ, Horne RW. The chemical composition and structure of the cell wall of Chlorella pyrenoidosa. Biochem J. 1958;70(3):391-7.

30. Allard B, Templier J. Comparison of neutral lipid profile of various trilaminar outer cell wall (TLS)-containing microalgae with emphasis on algaenan occurrence. Phytochemistry. 2000;54(4):369-80.

31. Atkinson AW, Gunning BES, John PCL. Sporopollenin in the cell wall of Chlorella and other algae: Ultrastructure, chemistry, and incorporation of 14C-acetate, studied in synchronous cultures. Planta. 1972;107(1):1-32.

32. Hibbs J. Anionic surfactants. In: Farn RJ, editor. Chemistry and technology of surfactants. Oxford: Blackwell Publishing; 2006. p. 91-132.

33. Zhang A, Wen X, Wang K, Huo Y, Geng Y, Ding Y, Li Y. Using surfactants for controlling rotifer contamination in mass cultivation of Chlorella pyrenoidosa. Algal Res. 2021;53:102166.

34. Scott MJ, Jones MN. The biodegradation of surfactants in the environment. Biochim Biophys Acta. 2000;1508(1):235-51.

35. Ding Y, Zhang A, Wen X, Wang Z, Wang K, Geng Y, Li Y. Application of surfactants for controlling destructive fungus contamination in mass cultivation of Haematococcus pluvialis. Bioresour Technol. 2020;317:124025.

36. Ding Y, Wen X, Peng X, Zhang A, Wang Z, Geng Y, Li Y. Surfactants as fungal parasite control agents in oleaginous microalga, Graesiella sp. WBG-1, mass culture. Algal Res. 2019;41:101539. 
37. Wen X, Du K, Wang Z, Peng X, Luo L, Tao H, Xu Y, Zhang D, Geng Y, Li Y. Effective cultivation of microalgae for biofuel production: a pilot-scale evaluation of a novel oleaginous microalga Graesiella sp. WBG-1. Biotechnol Biofuels. 2016;9(1):123.

38. Wen X, Geng Y, Li Y. Enhanced lipid production in Chlorella pyrenoidosa by continuous culture. Bioresour Technol. 2014;161:297-303.

39. Xie X, Huang A, Gu W, Zang Z, Pan G, Gao S, He L, Zhang B, Niu J, Lin A, Wang G. Photorespiration participates in the assimilation of acetate in Chlorella sorokiniana under high light. New Phytol. 2016;209(3):987-98.
40. Serra-Maia R, Bernard O, Gonçalves A, Bensalem S, Lopes F. Influence of temperature on Chlorella vulgaris growth and mortality rates in a photobioreactor. Algal Res. 2016;18:352-9.

\section{Publisher's Note}

Springer Nature remains neutral with regard to jurisdictional claims in published maps and institutional affiliations.
Ready to submit your research? Choose BMC and benefit from:

- fast, convenient online submission

- thorough peer review by experienced researchers in your field

- rapid publication on acceptance

- support for research data, including large and complex data types

- gold Open Access which fosters wider collaboration and increased citations

- maximum visibility for your research: over 100M website views per year

At BMC, research is always in progress.

Learn more biomedcentral.com/submissions 